\title{
Comparison of evaluations of hormone receptors in breast carcinoma by image-analysis using three automated immunohistochemical stainings
}

\author{
KOJI ARIHIRO, MIYO ODA, KATSUNARI OGAWA, KENSHI TOMINAGA, YOSHIE KANEKO, TOMOMI SHIMIZU, \\ SHIHO OHNISHI, MEGUMI ODA, YUKI KURITA, YUKO TAIRA, MASAYOSHI FUJII and MAIKO TANAKA
}

Department of Anatomical Pathology, Hiroshima University Hospital, Hiroshima 734-8551, Japan

Received June 22, 2010; Accepted August 6, 2010

DOI: $10.3892 /$ etm.2010.142

\begin{abstract}
The aim of this study was to compare the results of immunohistochemistry (IHC) assays evaluated by human examiners with the results evaluated by computerized image analysis, and to compare the computerized image analysis results among three automated IHC assays, namely the BioGenex, Dako and Ventana assays. All slides were semiquantitatively evaluated according to the Allred score and J-score by human examiners. The images were analyzed using MacSCOPE version 2.6 for Macintosh according to the $\mathrm{H}$-score and the percentage of positive-stained nuclei per area of carcinoma cells (PP) irrespective of the intensity of the stained nuclei. The H-score for the estrogen receptor (ER) was significantly correlated with the Allred score $(\mathrm{P}<0.0001)$ and the PP for the ER was significantly correlated with the J-score $(\mathrm{P}<0.0001)$, suggesting that the image analysis used in the present study is a useful method for the evaluation of ER status. Several discrepancies were identified between the Allred score and H-score and between the PP and J-score due to the positive-stained cytoplasm area of carcinoma cells and/ or the positive-stained nuclei area of non-carcinoma cells, including benign epithelial cells, lymphocytes and stromal cells. Accordingly, advances in the algorithm of the digitized analyzing system is necessary.
\end{abstract}

\section{Introduction}

Although a refined assessment of hormone receptors in breast carcinoma is necessary to select therapeutic agents, endocrine responsiveness has recently been defined as the presence of any detectable estrogen receptor (ER) according to the recommendations and thresholds for the post-operative

Correspondence to: Dr Koji Arihiro, Department of Anatomical Pathology, Hiroshima University Hospital, 1-2-3 Kasumi, Minami-ku, Hiroshima 734-8551, Japan

E-mail: arihiro@hiroshima-u.ac.jp

Key words: estrogen receptor, immunohistochemistry, automated staining, image analysis adjuvant systemic therapy of early breast cancer proposed by the St. Gallen International Expert Consensus meeting in 2009 (1). In other words, the previous three categories of endocrine responsiveness using 1 and 10\% cut-off values have been simplified, so that endocrine therapy is considered when any ER-positive cells are noted in the tumor. For the evaluation of hormone receptor (HR) status, it is recommended that the percentage of HR-positive cells be indicated on pathology reports rather than merely using scores. In particular, positivity for HRs of $50 \%$ or more of tumor cells is viewed as indicating highly endocrine-responsive tumors, suggesting that the HR must be reliably and accurately measured.

As to the immunohistochemistry (IHC) methods for the detection and quantification of the ER and progesterone receptor (PgR), the authors compared evaluations for HRs in breast carcinoma using two manual and three automated IHC assays, and showed intermethod variability indicated by multirater $\kappa$-values for the ER and $\mathrm{PgR}(\mathrm{ER}, \kappa=0.34 ; \mathrm{PgR}, \kappa=0.45)$ (2). In addition, to assess low levels of HR expression, the HR was evaluated by real-time monitoring polymerase chain reaction (RT-PCR) using complementary DNA produced by reverse transcription of the messenger RNA of each breast carcinoma. Although we showed an excellent correlation between RT-PCR results and those of the IHC method, there were some discrepancies between the results of RT-PCR and IHC due to the overestimation of HR-positive lymphocytes and mesenchymal cells in tumor stroma, among other factors (3).

To provide a standardized semi-quantitative measurement of the HR for IHC specimens, computerized image analysis has been employed since the late 1980s (4). These attempts were performed using various systems or software, including Cell Analysis System's CAS 100 (4), BIOCOM500 (5), CAS 200 (6), Image cytometry (7), Adobe Photoshop $(8,9)$, computer-supported analysis (10), SpectraCube ${ }^{\mathrm{TM}}(11)$, Chroma Vision Automated Cellular Imaging System (ACIS) (12-14), WinROOF (15), QCA (16) and VISUAL C ${ }^{++}$(17). In particular, automated image analysis technology, including AQUA (18), Ariol (14,19) and MatLab7 using digital image capturing (20), has recently been developed. Although it is emphasized that computerized image analysis has improved quantification, reproducibility and interobserver variability for the HR evaluation of breast carcinoma (4-20), the process 
of image analysis is known to be more time consuming and labor intensive than assessment by the human eye.

In Japan, there are three automated IHC methods approved by the Japanese Ministry of Health, Labor and Welfare to assess HR status in order to determine the suitability of endocrine treatment: automated IHC staining by Dako (Dako Corp., Glostrup, Denmark), BioGenex Corp. (San Ramon, CA, USA) and Ventana Medical Systems (Tucson, AZ, USA), each of which uses a different method for retrieving antigens and different types of antibodies or detection reagents. To date, no studies have directly compared these IHC computerized image analysis methods.

The aim of the present study was to assess the intermethod variability of these three IHC assays using image analysis. An additional aim was to find an optimal condition for image analysis that may be proposed as a reliable assay for ER determination.

\section{Materials and methods}

Samples. Fifty consecutive cases of invasive ductal carcinoma of the breast that had been surgically resected in 2004 and 2005 were selected from the files of the Department of Anatomical Pathology, Hiroshima University Hospital. H\&E-stained slides of each case were reviewed, and the presence of invasive carcinoma and adjacent non-neoplastic breast tissue was confirmed in all cases. The histological type of each tumor was invasive ductal carcinoma, not otherwise specified.

IHC assay for ER. From formalin-fixed, paraffin-embedded tissues, five $4-\mu \mathrm{m}$ sections were serially cut and mounted on pre-coated slides. IHC assays were carried out as described in previous reports $(2,3)$.

For IHC by the BioGenex system using an automated i6000 immunostainer (BioGenex Corp.), anti-ER mouse monoclonal antibody (mAb), ER88 (BioGenex Corp.) was used. Immunoperoxidase staining was performed according to the manufacturer's instructions (BioGenex Corp.).

For IHC by the Dako system using the Dako Autostainer ${ }^{\mathrm{TM}}$, anti-ER mAb, 1D5 (Dako Corp.) was used. Immunoperoxidase staining was performed according to the manufacturer's instructions (Dako Corp.).

For IHC by the Ventana system using the Ventana HX System BenchMark ${ }^{\mathrm{TM}}$ (Ventana Medical Systems), anti-ER mAb, 6F11 (Ventana Medical Systems) was used. All procedures were performed automatically in BenchMark ${ }^{\mathrm{TM}}$. Immunoperoxidase staining was performed according to the manufacturer's instructions (Ventana Medical Systems). Diaminobenzidine (DAB) was used as a chromogen substrate in all specimens. The sections were counterstained with hematoxylin.

Scoring system for human examiners. First, the presence or absence of staining of the nuclei of non-neoplastic ducts and acini in adjacent tissue was observed and was used as an internal control. The site for evaluation was not limited to the invasive area, but incorporated the entire lesion. Two scoring systems were used to evaluate the IHC findings, the Allred score $(21)$ and J-score $(2,3,22)$. The J-score comprises proportional values irrespective of the intensity of stained nuclei, and the proportion of cells stained in each specimen was recorded as 0 , none; $1,<1 \% ; 2,1-10 \% ; 3, \geq 10 \%$, as advocated and employed as the cut-off points in previous reports $(23,24)$.

All study specimens were scored by two different examiners (K.A. and M.O.) masked to the patient characteristics.

Computer-assisted digital analysis. Ten images were selected by pathologists and captured from each section at x200 magnification through a Hamamatsu C5810 color chilled 3CCD camera (Hamamatsu, Japan). The captured images were saved as JPEG images on the image analysis computer. When contamination by normal tissue was present, the pathologist exchanged it manually for another field, including the tumor area. The images were analyzed using MacSCOPE version 2.6 (Mitani Corp., Tokyo, Japan) for Macintosh. Despite the area selection, a certain minimum contamination of the automatic measurement by host cells is inevitable when stromal and normal cells are intimately associated with tumor cells. To distinguish non-carcinoma cell elements from the nonimmunostained carcinoma cell nuclei on the digitized image, nuclei with small areas $\left(<25 \mu \mathrm{m}^{2}\right.$ gross area) and spindle features $(>0.5$ oval rate) were regarded as lymphocyte nuclei or nucleic debris and stromal cell nuclei, respectively, and were eliminated. Nuclei that stained brown or blue were extracted automatically using two distinct macroinstructions composed chiefly of algorithms for color extraction based on red-greenblue (RGB) parameters divided into 256 arbitrary units. To create the digitized image-based procedures for determining ER status, the threshold value of the RGB parameters of each intensity score (IS) was established as: 0 , negative; 1 , weak nuclear staining, faintly perceptible at high power magnification; 2 , intermediate stained nuclei; 3 , nuclei displaying strong staining that had the appearance of an ink dot at low power magnification, according to the Allred score (Fig. 1).

Scoring system. Two scoring systems were used to evaluate the ER findings using computer-assisted analysis: the H-score method $(15,25)$ and the percentage of area of stained nuclei of carcinoma cells (PP) in 10 images, irrespective of the intensity of stained nuclei. The $\mathrm{H}$-score was calculated by summing $3 \mathrm{x}$ the percentage of total nuclei area showing IS3, $2 x$ the percentage of total nuclei area showing IS2 and 1x the percentage of total nuclei area showing IS1, ranging from 0 to 300 .

Statistical analysis. The Spearman's rank correlation test was used for correlation analysis between the $\mathrm{H}$-score and the total score (TS) of the Allred score and between the PP and J-score.

\section{Results}

Relationship between Allred score and H-score for ER. A comparison of the distribution of the TS of Allred scores determined by the human examiners and that of $\mathrm{H}$-scores calculated by image-analysis software for ER by the three staining methods is shown in Fig. 2. The H-score values for the same group of tumors increased monotonically as the TS increased, although there was considerable variability among tumors with the same TS. The Spearman's rank correlation coefficient between the two methods ranged from 0.572 to $0.889(\mathrm{P}<0.0001)$. The cut-off values of $\mathrm{H}$-scores for the ER 

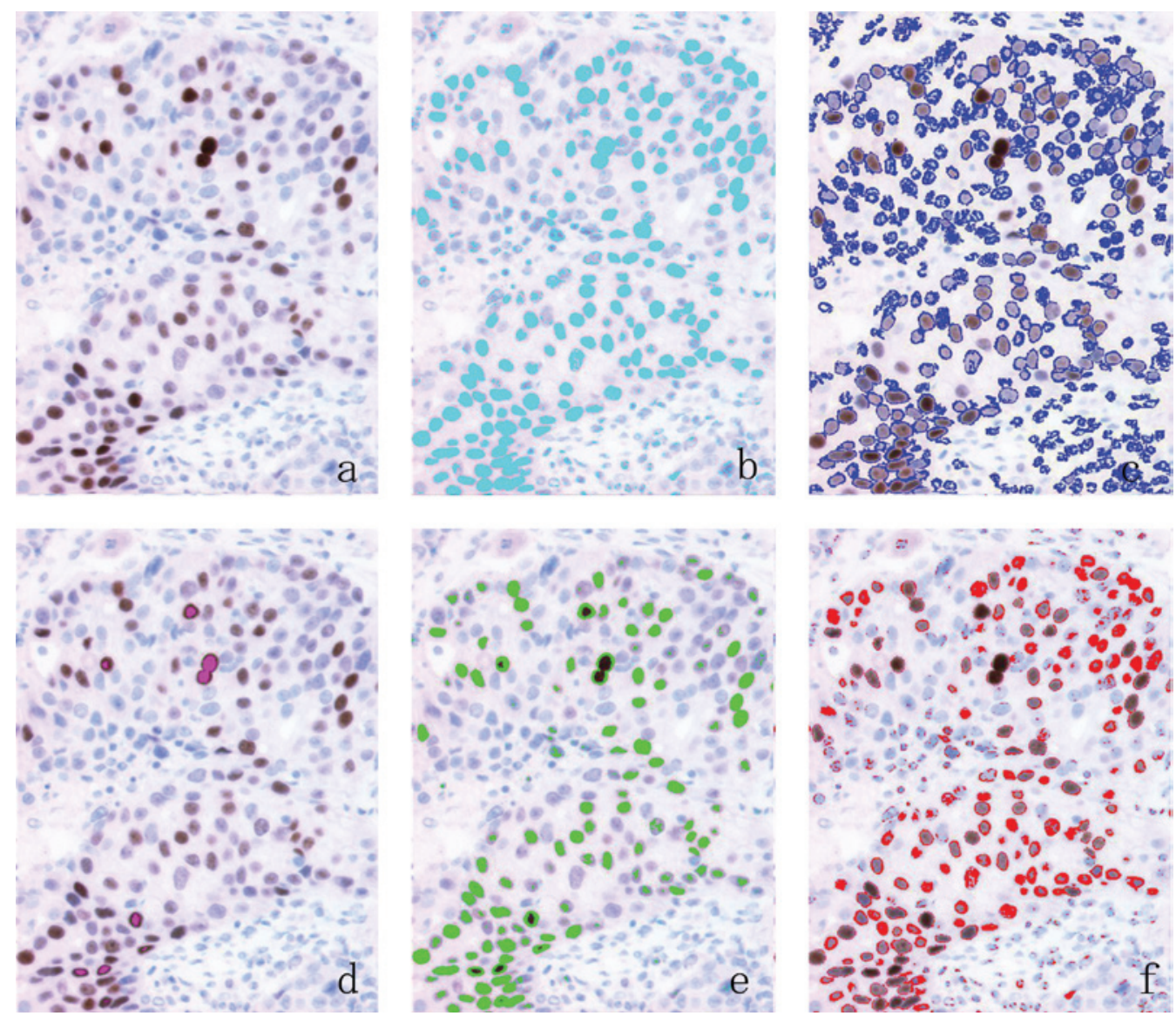

Figure 1. Immunohistochemistry of ER and the images analyzed by MACScope. (a) Nuclei of carcinoma cells show various intensities of the ER. (b) All positive-stained nuclei of carcinoma cells are shown in light blue; 3,994.4 $\mu \mathrm{m}^{2}$. (c) All negative-stained nuclei of carcinoma cells are shown in blue; $5,409.3 \mu \mathrm{m}^{2}$. (d) Strongly stained nuclei of carcinoma cells are shown in pink; $92.6 \mu \mathrm{m}^{2}$. (e) Intermediately stained nuclei of carcinoma cells are shown in green; $1,728.9 \mu \mathrm{m}^{2}$. (f) Weakly stained nuclei of carcinoma cells are shown in red; $2,173 \mu \mathrm{m}^{2}$.

Dako

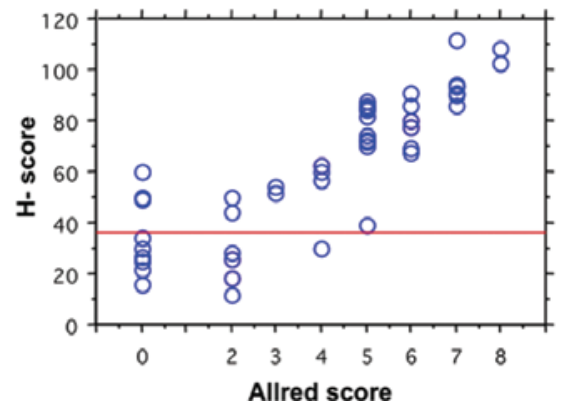

Ventana

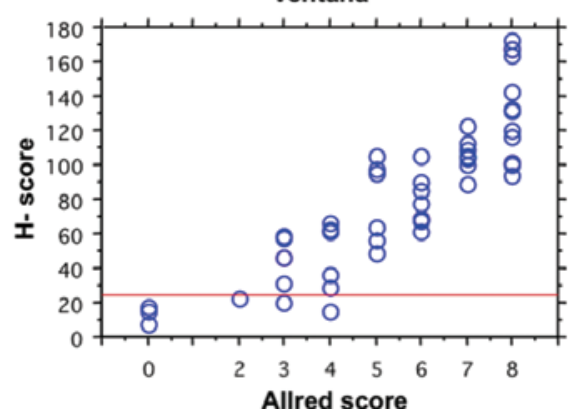

BioGenx

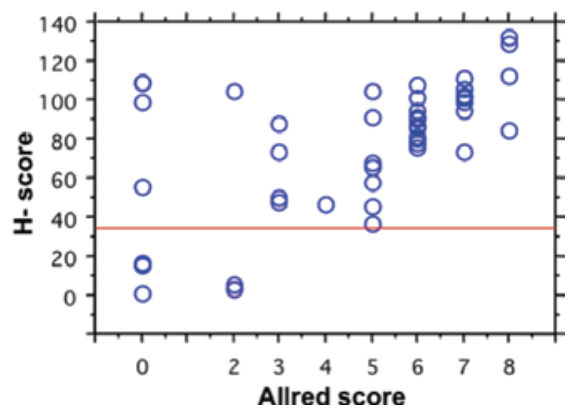

Figure 2. Scattergram of H-score with Allred score for the ER. The H-score was significantly correlated to the Allred total score of IHC using the Dako, Ventana and BioGenex assays (Spearman's rank correlation test, $\mathrm{P}<0.0001$ ). The cut-off values in the Dako, Ventana and BioGenex assays were 36,24 and 34 (red line), respectively.

determined by the Dako, Ventana and BioGenex assays were regarded as 36, 24 and 34 according to the Allred score, respectively. The concordance rates at these cut-off values were the highest of each IHC assay, and 88, 98 and 90\% among the IHC results generated by the Dako, Ventana and BioGenex assays, respectively.

Relationship between J-score and PP for ER. A comparison of the distribution of J-scores scored by the human examiners and that of the percentage of positive cells calculated by image-analyzing software for ER by the three staining methods is shown in Fig. 3. The Spearman's rank correlation coefficient between the two methods ranged from 0.495 to 0.914. The cut-off values of the PP for ER determined by the Dako, Ventana and BioGenex assays were regarded as 37,36 and 38 according to the J-score, respectively. The concordance rates at these cut-off values were the highest of each IHC assay, and 90, 92 and 84\% among the IHC results generated by the Dako, Ventana and BioGenex assays, respectively. 

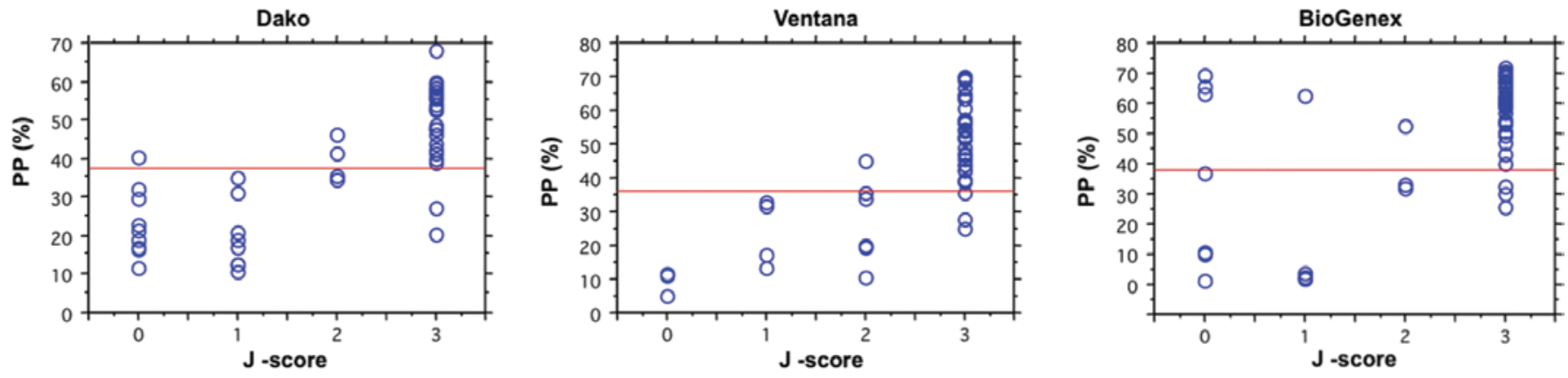

Figure 3. Scattergram of PP with J-score for the ER. The PP is significantly correlated to the J-score for the ER using the Dako, Ventana and BioGenex assays (Spearman's rank correlation test, $\mathrm{P}<0.0001$ ). The cutoff values in the Dako, Ventana and BioGenex assays were 37,36 and 38 (red line), respectively.
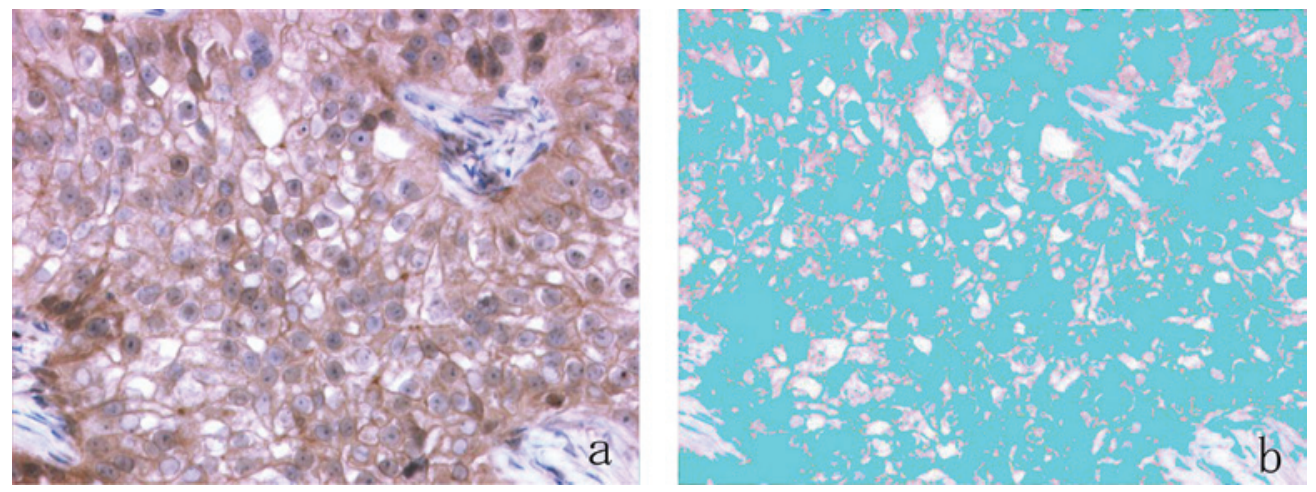

Figure 4. Immunohistochemistry of the ER and the images analyzed by MACScope. (a) ER positivity was noted not only in the nuclei, but also in the membrane and/or cytoplasm of carcinoma cells. (b) All positive-stained areas of carcinoma cells are shown in light blue. ER status was overestimated.

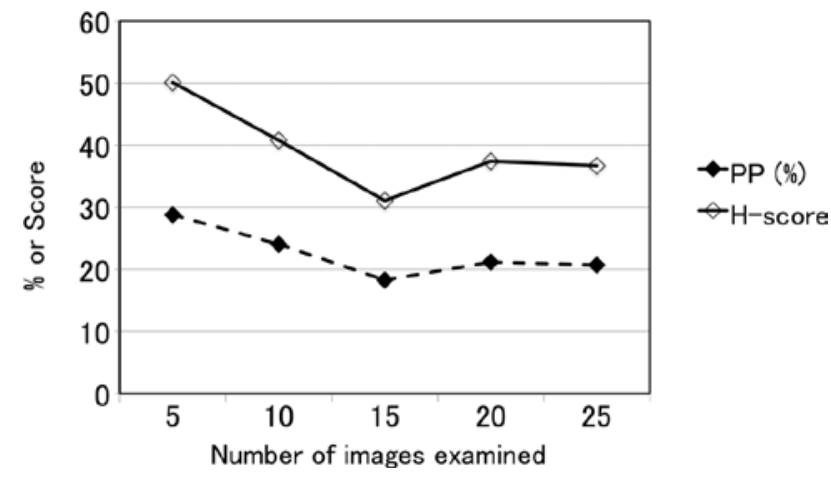

Figure 5. Correlation between numbers examined by computerized image analysis, $\mathrm{H}$-score and the $\mathrm{PP}$ of $\mathrm{ER}$ in a representative case. The $\mathrm{H}$-score and $\mathrm{PP}$ show a plateau in more than 20 images examined.

Procedures. Approximately 4 min were required to capture 10 images from each specimen and to process these image analyses per case. The individual results of 50 cases of breast carcinoma in the present study were transferred to another software program for calculation.

\section{Discussion}

In the present study, quantification of ER using imageanalyzing software was performed and compared to semi-quantitative assessment by histopathologists. A high degree of correlation between the two was revealed. Although various studies concerning computerized image analysis have been conducted (4-25), it is difficult to compare the various systems directly, due to the absence of reliable and universal gold standards.

With regard to a cut-off value for ER evaluation by computerized image analysis, $10 \%$ of positive cells $(9,16,20)$, the H-score (15) and AQUA scores calculated by the average signal intensity divided by compartment area (18) were used in previous reports. Although in the present study the Allred score determined by human examiners was compared to the $\mathrm{H}$-score calculated by the computer and the Allred/H-score conversion table was shown in a previous report (26), it was difficult to translate one scoring system to the other system, precisely since the two systems are not strictly equivalent. Accordingly, the final assessment of the clinical usefulness of these various systems, cut-off values and estimation methods is thought to depend on the correlation with biological behavior and responsiveness to hormone therapy.

In the present study, there were some discrepancies between human observation and computerized image analysis for IHC. For the cases showing negativity on human observation and positivity on computerized image analysis for ER, the software used detected ER expression in the lymphocytes and mesenchymal cells. Since ER expression of infiltrating lymphocytes and mesenchymal cells in tumor stroma has been reported previously $(3,27)$, a condition was set to eliminate non-carcinoma cells showing positivity, although it was not possible to completely eliminate the ER expression of lymphocytes or mesenchymal cells. 
Additionally, in the present study, ER expression in the cytoplasm or plasma membrane of carcinoma cells was noted in 3.5, 2 and 25\% of examined cases using the Dako, Ventana and BioGenex assays, respectively (Fig. 4). In a previous study, the extranuclear expression of HR in breast carcinoma cells was reported in $9.5 \%$ of examined cases (28). Accordingly, the software used in the present study may have overestimated the positivity of ER in carcinoma cells, as it could not discriminate between ER expression in the nuclei and that in the cytoplasm or plasma membrane of carcinoma cells. Accordingly, it is necessary to use softwave with improved function that derives ER expression only from the nuclei of carcinoma cells.

Regarding the time required to perform computerized image analysis, in general, the process of image analysis is more time consuming and labor intensive than visual scoring from a glass slide. Although in a previous report the processing time for 100 images from 20 cases by WinROOF was reportedly approximately $60 \mathrm{~min}$, excluding the time required to capture the images (15), there have been few reports discussing the time necessary to capture and process the images by software. In the present study, the capture and processing time for 10 images per case was approximately $4 \mathrm{~min}$. In comparison to the study using WinROOF, the process of image analysis in the present study was less time consuming and labour intensive due to the improved function of the computer and software.

With regard to the number of images captured for digitized analysis, there have been various numbers of images used ranging from the single best field $(16,17)$, three fields (8), four fields (14), five fields $(5,15,29)$, eight fields (9) and ten fields $(6,10)$. In the present study, ten fields were selected and captured for image analysis, similar to previous studies which used the maximal numbers of images $(6,10)$. Although twenty fields was regarded as sufficient for digitized image analysis on the basis of a preliminary study on the relationship between the number of images digitally analyzed and H-score or the percentage of stained nuclei area (Fig. 5), the optimal number of images for digital analysis should be considered, taking into account the time required for the procedure. Recently, a fully automatic digitized analyzing system for the total fields of specimens was developed using digital images captured by the Aperio ScanScope XT Slide Scanner and algorithm by MatLab 7 (20). This system reportedly identifies only tumor nuclei and automatically excludes non-tumor structures, including stromal components and lymphocytes. In particular, it is entirely unsupervised and does not require any a priori data. Irrespective of the adjustment of various thresholds and cut-off values to detect various cells exhibiting particular sizes and shapes, to date, it has been difficult for a digitized analyzing system to discriminate between benign and malignant cells with complete accuracy. Accordingly, advances in the algorithm of digitized analyzing systems are necessary.

As for image analysis of the RGB system, in general, a composite color signal is built up from combinations of basic color values produced by the mosaic arrangement of three color filters (red, green and blue) on the surface of the imager. In this way, each color image is recorded as a superimposition of 3 images with a photometric resolution of 255 linear values: a red, a blue and a green one, reflecting the slide trans- mission into these three types of wavelengths (5). However, even when an RGB imaging system functions perfectly, there are intrinsic limitations to its ability to distinguish between similar chromogens and to isolate the optical signal from each chromogen. Thus, each color signal is quantitatively and separately measured (30). In the present study, DAB was used as a chromogen substrate, and the choice of chromogen and counterstain is known to affect both the visual and quantitative results. Accordingly, the appropriate chromogen substrate and counterstain dye suitable for image analysis must be selected.

\section{References}

1. Goldhirsch A, Ingle JN, Gelber RD, Coates AS, Thurlimann B and Senn HJ: Thresholds for therapies: highlights of the St Gallen International Expert Consensus on the primary therapy of early breast cancer 2009. Ann Oncol 20: 1319-1329, 2009.

2. Arihiro K, Umemura S, Kurosumi M, et al: Comparison of evaluations for hormone receptors in breast carcinoma using two manual and three automated immunohistochemical assays. Am J Clin Pathol 127: 356-365, 2007.

3. Oda M, Arihiro K, Kataoka T, Osaki A, Asahara T and Ohdan H: Comparison of immunohistochemical assays and reverse transcription real-time polymerase chain reaction for analyzing status of hormone receptors in human breast carcinoma. Pathol Int 60: 305-315, 2010.

4. Bacus S, Flowers JL, Press MF, Bacus JW and McCarty KS Jr: The evaluation of estrogen receptor in primary breast carcinoma by computer-assisted image analysis. Am J Clin Pathol 90: 233-239, 1988

5. Rostagno P, Birtwisle I, Ettore F, et al: Immunohistochemical determination of nuclear antigens by colour image analysis: application for labelling index, estrogen and progesterone receptor status in breast cancer. Anal Cell Pathol 7: 275-287, 1994.

6. Layfield LJ, Saria EA, Conlon DH and Kerns BJ: Estrogen and progesterone receptor status determined by the Ventana ES 320 automated immunohistochemical stainer and the CAS 200 image analyzer in 236 early-stage breast carcinomas: prognostic significance. J Surg Oncol 61: 177-184, 1996.

7. Cohen C: Image cytometric analysis in pathology. Hum Pathol 27: 482-493, 1996.

8. Lehr HA, Mankoff DA, Corwin D, Santeusanio G and Gown AM: Application of photoshop-based image analysis to quantification of hormone receptor expression in breast cancer. J Histochem Cytochem 45: 1559-1565, 1997.

9. Bejar J, Sabo E, Misselevich I, Eldar S and Boss JH: Comparative study of computer-assisted image analysis and light-microscopically determined estrogen receptor status of breast carcinomas. Arch Pathol Lab Med 122: 346-352, 1998.

10. Mofidi R, Walsh R, Ridgway PF, et al: Objective measurement of breast cancer oestrogen receptor status through digital image analysis. Eur J Surg Oncol 29: 20-24, 2003.

11. Rothmann C, Barshack I, Gil A, Goldberg I, Kopolovic J and Malik Z: Potential use of spectral image analysis for the quantitative evaluation of estrogen receptors in breast cancer. Histol Histopathol 15: 1051-1057, 2000.

12. Vesoulis Z, Rajappannair L, Define L, Beach J, Schnell B and Myers S: Quantitative image analysis of estrogen receptors in breast fine needle aspiration biopsies. Anal Quant Cytol Histol 26: 323-330, 2004.

13. Fisher ER, Anderson S, Dean S, et al: Solving the dilemma of the immunohistochemical and other methods used for scoring estrogen receptor and progesterone receptor in patients with invasive breast carcinoma. Cancer 103: 164-173, 2005.

14. Gokhale S, Rosen D, Sneige N, et al: Assessment of two automated imaging systems in evaluating estrogen receptor status in breast carcinoma. Appl Immunohistochem Mol Morphol 15: 451-455, 2007

15. Hatanaka Y, Hashizume K, Nitta K, Kato T, Itoh I and Tani Y: Cytometrical image analysis for immunohistochemical hormone receptor status in breast carcinomas. Pathol Int 53: 693-699, 2003.

16. Diaz LK, Sahin A and Sneige N: Interobserver agreement for estrogen receptor immunohistochemical analysis in breast cancer: a comparison of manual and computer-assisted scoring methods. Ann Diagn Pathol 8: 23-27, 2004. 
17. Sharangpani GM, Joshi AS, Porter K, et al: Semi-automated imaging system to quantitate estrogen and progesterone receptor immunoreactivity in human breast cancer. J Microsc 226: 244-255, 2007.

18. Chung GG, Zerkowski MP, Ghosh S, Camp RL and Rimm DL: Quantitative analysis of estrogen receptor heterogeneity in breast cancer. Lab Invest 87: 662-669, 2007.

19. Turbin DA, Leung S, Cheang MC, et al: Automated quantitative analysis of estrogen receptor expression in breast carcinoma does not differ from expert pathologist scoring: a tissue microarray study of 3,484 cases. Breast Cancer Res Treat 110: 417-426, 2008.

20. Rexhepaj E, Brennan DJ, Holloway P, et al: Novel image analysis approach for quantifying expression of nuclear proteins assessed by immunohistochemistry: application to measurement of oestrogen and progesterone receptor levels in breast cancer. Breast Cancer Res 10: R89, 2008.

21. Allred DC, Harvey JM, Berardo M and Clark GM: Prognostic and predictive factors in breast cancer by immunohistochemical analysis. Mod Pathol 11: 155-168, 1998.

22. Umemura S, Kurosumi M, Moriya T, et al: Immunohistochemical evaluation for hormone receptors in breast cancer: a practically useful evaluation system and handling protocol. Breast Cancer 13: 232-235, 2006.

23. Goldhirsch A, Glick JH, Gelber RD, Coates AS, Thurlimann B and Senn HJ: Meeting highlights: international expert consensus on the primary therapy of early breast cancer 2005. Ann Oncol 16: $1569-1583,2005$.
24. Kaplan PA, Frazier SR, Loy TS, Diaz-Arias AA, Bradley K and Bickel JT: 1D5 and 6F11: an immunohistochemical comparison of two monoclonal antibodies for the evaluation of estrogen receptor status in primary breast carcinoma. Am J Clin Pathol 123: 276-280, 2005.

25. Goulding H, Pinder S, Cannon $\mathrm{P}$, et al: A new immunohistochemical antibody for the assessment of estrogen receptor status on routine formalin-fixed tissue samples. Hum Pathol 26: 291-294, 1995.

26. Shousha S: Oestrogen receptor status of breast carcinoma: Allred/H score conversion table. Histopathology 53: 346-347, 2008.

27. Sapino A, Cassoni P, Ferrero E, et al: Estrogen receptor alpha is a novel marker expressed by follicular dendritic cells in lymph nodes and tumor-associated lymphoid infiltrates. Am J Pathol 163: 1313-1320, 2003

28. Kim R, Kaneko M, Arihiro K, et al: Extranuclear expression of hormone receptors in primary breast cancer. Ann Oncol 17: 1213-1220, 2006

29. Kostopoulos S, Glotsos D, Cavouras D, et al: Computer-based association of the texture of expressed estrogen receptor nuclei with histologic grade using immunohistochemically-stained breast carcinomas. Anal Quant Cytol Histol 31: 187-196, 2009.

30. Taylor CR and Levenson RM: Quantification of immunohistochemistry - issues concerning methods, utility and semiquantitative assessment II. Histopathology 49: 411-424, 2006. 